\title{
Factores que influyen \\ en el aprendizaje del \\ inglés de los bachilleres \\ de Pasto, Colombia
}

Factors

Influencing

English Language

Learning of High

School Students

in Pasto, Colombia

\author{
Fatores que \\ influenciam 0 \\ aprendizado \\ de inglês de \\ estudantes do \\ ensino médio em \\ Pasto, Colômbia
}

\section{Para citar este artículo}

Bastidas, J. y Muñoz, G. (2020). Factores que influyen en el aprendizaje del inglés de los bachilleres de Pasto, Colombia. Folios, 51, 163-181. doi: 10.17227/folios.51-8676

Artículo recibido $08 \cdot 11 \cdot 2018$

Artículo aprobado $25 \cdot 07 \cdot 2019$

* PhD in Language, Literacy \& Learning, University of Southern California, Los Angeles, CA, USA. Profesor del Departamento de Lingüística e Idiomas, Universidad Pedagógica Nacional, Universidad de Nariño, Pasto, Nariño. Correo electrónico: jabas3@udenar.edu.co

** Magister en Didáctica del Inglés, Manizales, Caldas, Colombia. Profesora del Departamento de Lingüística e Idiomas, Universidad de Nariño, Pasto, Nariño.

Correo electrónico: gabymib@udenar.edu.co 


\section{Resumen}

En la literatura de la Adquisición de una Segunda Lengua (ASL), campo desarrollado en contextos donde la lengua se utiliza como medio de comunicación, y de Aprendizaje de una Lengua Extranjera (ALE), subcampo en el que la lengua se utiliza fundamentalmente como asignatura de estudio en las aulas de clase, se han identificado varios factores que contribuyen a su aprendizaje. Para avanzar en esta línea de investigación sobre factores que inciden en el aprendizaje de las lenguas, se diseñó un estudio sustentado en un paradigma empírico-analítico y en un método descriptivo, cuyo objetivo general fue analizar los factores internos y externos que tienen una mayor influencia sobre el nivel de aprendizaje del inglés como lengua extranjera (AILE), según las percepciones de los estudiantes y profesores de bachillerato de Pasto. Los datos fueron recogidos por medio de dos cuestionarios, aplicados a 113 estudiantes recién egresados de bachillerato y a 25 profesores. Los resultados indicaron que según las percepciones de los estudiantes y de los profesores, tanto factores externos como internos contribuyen positivamente en el aile. Sin embargo, los factores externos que parecen contribuir más se relacionan, por una parte, con 1) el ambiente del aprendizaje en el aula, 2) el currículo institucional, 3) la preparación profesional de los profesores, 4) el papel de la comunidad local y 5) el papel de las políticas nacionales. Por otra parte, mientras los profesores resaltaron los factores internos de carácter cognitivo y afectivo, los estudiantes destacaron los factores cognitivos y lingüísticos.

\section{Palabras clave}

aprendizaje de las lenguas extranjeras; contexto social; cognición-emoción; inglés como lengua extranjera; educación secundaria

Abstract

The review of the literature related to Second Language Acquisition and Foreign Language Learning has shown several factors that promote their learning process, both in natural and academic contexts. In order to advance the area of factors affecting second or foreign language learning, the researchers have conducted a study based on an empirical-analytical paradigm and a descriptive method, whose general objective was "to analyze the internal and external factors which might have a major influence on the level of English as a foreign language learning (EFLL), according to the perceptions of students and teachers of secondary school in Pasto". The data was gathered through two questionnaires applied on 113 students who have just graduated from high school and 25 teachers. The results indicated that both external and internal factors impact positively to EFLL, according to the students' and teachers' perceptions. However, the most relevant external factors are related, on the one hand, to 1) the learning environment in the classroom, 2) the institutional curriculum, 3) the teachers' professional preparation, 4) the role of the local community, and 5) the role of national educational policies. On the other hand, while the teachers stood out the cognitive and affective internal factors, the students highlighted the cognitive and linguistic factors.

\footnotetext{
Keywords

foreign language learning; social context; cognition-emotion; English as a foreign language; secondary education
}

\section{Resumo}

Na literatura da Aquisição de uma segunda língua (ASL), um campo desenvolvido em contextos em que a linguagem é usada como meio de comunicação e de Aprendizagem de uma língua estrangeira (ALE), um subcampo no qual a linguagem é usada principalmente como objeto de estudo nas salas de aula, vários fatores que contribuem para sua aprendizagem foram identificados. Para avançar nessa linha de pesquisa sobre fatores que afetam o aprendizado de idiomas, foi elaborado um estudo baseado em um paradigma empíricoanalítico e em um método descritivo, cujo objetivo geral era analisar fatores internos e externos que exercem maior influência sobre o nível de aprendizagem de inglês como língua estrangeira (AILE), de acordo com as percepções de alunos e professores do ensino médio de Pasto. Os dados foram coletados por meio d; dois questionários, aplicados a 113 alunos recém-formados do ensino médio e 25 professores. Os resultados indicaram que, de acordo com as percepções de alunos e professores, fatores externos e internos contribuem positivamente na AlLE. No entanto, fatores externos que parecem contribuir mais estão relacionados, por um lado, a 1) 0 ambiente de aprendizagem em sala de aula, 2) o currículo institucional, 3) a preparação profissional dos professores, 4) o papel da comunidade local e 5) o papel das políticas nacionais. Por outro lado, enquanto os professores destacaram fatores cognitivos e afetivos internos, os alunos destacaram fatores cognitivos e linguísticos.

\section{Palavras-chave}

aprendizagem de línguas estrangeiras; contexto social; cognição-emoção; Inglês como língua estrangeira; ensino médio

\section{- 164}




\section{Introducción}

Los avances y cambios de tipo social, político, económico, cultural, científico, educativo y tecnológico en el mundo han contribuido a sustentar el estudio de la lengua inglesa en calidad de lengua franca que facilita la comunicación internacional. En consecuencia, el número de personas que estudian este idioma como lengua internacional se incrementa anualmente, a nivel mundial. Por ejemplo, según la compañía English First (EF, 2016) en la presente década se proyecta que alrededor de 2.000 millones de personas estudiarán inglés. Sin embargo, a pesar de esta tendencia, los resultados de aprendizaje efectivo de los adultos egresados de secundarias que voluntariamente toman la prueba EF-EPI, provenientes de algunas regiones de mundo como los países de Medio Oriente, África, Latinoamérica y varios países de Asia se ubicaron (en promedio) en las bandas entre muy bajo (A1 según el Marco Común Europeo de Referencia, MCER, 2001) y bajo (A2). Los resultados de Colombia no son la excepción, ya que en las pruebas de 2016 Colombia se ubicó en el puesto 51 (de 80 países) con un puntaje de 49.97 que corresponde al nivel bajo (EF, 2017). Estos resultados están en concordancia con aquellos de las pruebas diagnósticas del Plan Nacional de Bilingüismo (PNB), realizadas entre 2003 y 2006 y los resultados de las pruebas SABER 11, que han sido aplicadas por el ICFES entre 2007 y 2017, los cuales indican que la mayoría de los bachilleres se ubican entre los niveles -A1 y A2, según las bandas de dicha prueba (Alonso y otros, 2016; Bastidas y Muñoz, 2011; Bastidas y Muñoz, 2018 y Sánchez, 2013). Es decir, que el fenómeno del bajo nivel de aprendizaje del inglés de los bachilleres colombianos, incluidos los de Pasto, sigue siendo persistente, tal como se reportó en Bastidas y Muñoz (2018).

Para obtener un mayor entendimiento de esta problemática, que no es solo nacional, sino también internacional, es necesario recurrir a la revisión de la literatura sobre el campo del aprendizaje y la enseñanza del inglés. Esta revisión se concentra en el área específica de factores externos e internos que parecen afectar el proceso de aprendizaje, tanto en los contextos internacionales del Aprendizaje del Inglés como Segunda Lengua (AISL), como en los contextos internacionales y nacionales del Aprendizaje del Inglés como Lengua Extranjera (AILE).

En primer lugar, los estudios sobre factores internos del aprendiz que más se han realizado han sido en el campo de ASL, dado su interés en la descripción y explicación del proceso de aprendizaje, tanto en contextos naturales como instruccionales, donde la L2 se usa en la comunidad circundante para propósitos de interacción y comunicación entre sus hablantes. Según autores como Brown (2007), Ellis (1994), Ellis (2015), Larsen-Freeman \& Long (1991) y Sanz (2005), los factores que se han identificado como aquellos que se correlacionan o demuestran que pueden afectar el proceso de ASL en una gran cantidad de investigaciones son de carácter biológico, cognitivo, afectivo, lingüístico y psico-socioafectivo. Entre los factores biológicos se destacan la edad y el género de los aprendices. Los factores de tipo cognitivo bastante estudiados han sido los estilos de aprendizaje, las estrategias de aprendizaje, la aptitud para aprender una L2 y en menor escala la atención y la memoria de trabajo (Sanz, 2005). Los factores afectivos que han recibido mayor atención han sido la ansiedad, la autoestima, la extroversión y la introversión, la empatía, la inhibición y el asumir riesgos, y con menor énfasis, la tolerancia a la ambigüedad, el temor al rechazo y la autoeficacia. Entre los factores lingüísticos que Brown (2007) destaca tenemos: errores, fosilización de errores, transferencia, interferencia e interlengua. Finalmente, los factores que también se han destacado por su correlación y efecto sobre el aprendizaje son los de carácter psico-socioafectivo como la motivación y la actitud hacia el AsL.

Aunque el énfasis de la investigación en el campo de ASL ha sido sobre los factores internos, algunos autores también reportan investigaciones sobre la incidencia de tipo social, sociocultural y pedagógico. Sin embargo, según Ellis (1994) estos factores posiblemente no influyen sobre el aprendizaje directamente, sino a través de la mediación de otras 
variables, entre ellas la actitud hacia el aprendizaje. Podríamos, también agregar que las percepciones, las creencias, las motivaciones y los estados afectivos de los aprendices pueden incrementar o contrarrestar los efectos de los factores externos antes indicados.

Entre los factores socioculturales que destaca Brown (2007) tenemos: clase social, identidad étnica, estereotipos culturales, cultura de la L2 y distancia social. Por su parte, Ellis (1994) y Sanz (2005) se refieren a los siguientes factores provenientes del contexto social, tanto natural como instruccional: calidad de la lengua a la que se expone y procesa el aprendiz (en inglés: language input), interacción, errores y su retroalimentación y producción lingüística (en inglés: language output). Finalmente, Ellis (1994) y Sanz (2005) se refieren a la diversidad de investigaciones sobre intervenciones pedagógicas en el aula de clase, las cuales han identificado una serie de factores, tales como: input, actividades de práctica de la L2, efecto de los métodos de enseñanza, interacción, efecto de la enseñanza formal de los componentes (sonidos, léxico y gramática) y de las habilidades lingüísticas (escucha, habla, lectura y escritura), calidad de la expresión oral en L2 del profesor, tratamiento de los errores, preguntas del profesor, participación del estudiante, tipos de tareas o actividades, trabajo cooperativo y otras. Aunque la tendencia es reconocer que algunas intervenciones pedagógicas facilitan el desarrollo natural de la L2, que ciertas actividades que se centran en el significado en un contexto comunicativo producen buenos resultados de aprendizaje (Ellis, 1994) y que varios estudios de tipo cuasiexperimental y experimental han demostrado cierta efectividad de la instrucción formal sobre el aprendizaje de una L2 (Norris y Ortega, 2000), los investigadores de este campo no se atreven a afirmar en forma contundente que dichas intervenciones contribuyan al aprendizaje efectivo de una segunda lengua.

En el campo del AILE, también se han realizado una gran cantidad de investigaciones. En este apartado nos referimos a los resultados de las investigaciones específicas sobre factores que afectan el aprendizaje del inglés de la escuela secundaria. Los estudios publicados en revistas internacionales han encontrado correlaciones o explicaciones entre factores internos y el nivel de AILE. Entre los factores estudiados tenemos: de carácter biológico como la edad (Madrid, 1980) y el género (Nyikos, 1990; Zoghi et ál; 2013); cognitivos como la motivación (Alsayed, 2003; Dörnyei y Csizer, 1998; León, 2013; López, Quesada y Salas, 2014; Madrid et ál., 1993), efecto de los conocimientos previos del inglés y dificultad para aprenderlo (Souriyavongsa et ál., 2013); y de carácter afectivo como miedo a cometer errores (Souriyavongsa et ál., 2013).

Por otra parte, los resultados de las investigaciones demuestran la existencia de varios factores externos de tipo contextual que se correlacionan o explican el rendimiento académico de los estudiantes de secundaria, medido a través de pruebas estandarizadas o pruebas de logro académicas de carácter institucional. Entre estos factores tenemos: clase social (Madrid, 1995); factores socioeconómicos (López, Quesada y Salas, 2014; Misbah et ál., 2017) y aprendizaje temprano del inglés (Alsayed, 2003). También se destacan los factores de tipo instruccional como: poca preparación de los profesores, tanto en inglés como en metodología de la enseñanza (León, 2013; Quesada et ál., 2017; Souriyavongsa et ál., 2013) currículo inapropiado (Souriyavongsa et ál, 2013), falta de recursos instruccionales y grupos numerosos (León, 2013).

Finalmente, la revisión de los estudios que se han realizado sobre el nivel de inglés de los bachilleres colombianos nos ha permitido clasificarlos en estudios históricos (Bastidas, 2017; Bastidas y Muñoz, 2017; Gómez, 1971; Hidalgo y Caicedo, 2017); revisión de tema (Quintanilla, 1971) y estudios de investigación empírica (Avella y Camargo, 2010; Bastidas, 1991; Bastidas y Muñoz, 2018; Cardona y Hanley, 1985; Hernández y Faustino, 2006; Navarrete, 1970; Stansfield, 1972). Los anteriores estudios, en mayor o menor frecuencia, coinciden en resaltar los factores de tipo sociocultural y educativo sobre los factores internos al estudiante. Lo anterior se podría explicar por la presencia de una cantidad 
de factores que rodean al estudio del inglés en un contexto extranjero, cuyo centro de aprendizaje es el aula de clase.

El estudio de Bastidas y Muñoz (2018), cuyo propósito fue "comprender el fenómeno del bajo nivel de aprendizaje del inglés de los bachilleres, según las percepciones de estudiantes (actuales y egresados) y profesores de un grupo selecto de colegios de Pasto, Colombia" (p. 7) desde un paradigma interpretativo nos permitió descubrir cinco categorías: ambiente de aprendizaje en el aula (AAA), currículo institucional (CI), papel de la comunidad local (PCL), papel de las políticas educativas nacionales (PEN) y papel de la comunidad internacional (PCI). Estas categorías fueron utilizadas para la elaboración de los instrumentos cuantitativos que se utilizaron en la presente investigación, con el objeto de identificar aquellos factores, que no solamente fuesen percibidos por los estudiantes y los profesores, como los que inciden en el bajo nivel de aprendizaje, sino también que contribuyen al aprendizaje efectivo del inglés en el contexto escolar en el estudio de Bastidas y Muñoz (2018).

En las categorías de AAA y CI, Bastidas y Muñoz (2018) coinciden con otros autores en la identificación de factores relacionados con una metodología de enseñanza basada en métodos tradicionales, tales como el Método de Gramática y Traducción, el Método Directo y el Método AudioLingue (Bastidas, 1991; Bastidas, 2017; Bastidas y Muñoz, 2017; Bastidas y Muñoz, 2018; Gómez, 1971; Navarrete, 1970); con un grupo de profesores insuficientemente preparados, especialmente en el manejo del inglés, con una metodología de enseñanza inapropiada y con el uso inadecuado de los libros de texto. En lo institucional, la mayor parte de autores se refieren a los grupos numerosos y heterogéneos, a la baja intensidad horaria semanal, a la inexistencia o insuficiencia de materiales instruccionales, tales como libros de texto y ayudas audiovisuales, a la planeación inadecuada del área de inglés, al cambio frecuente de profesores de un nivel a otro, a su consecuente desarticulación y falta de continuidad y a la falta de apoyo, tanto al área de inglés en el currículo institucional, como a la capacitación y actualización de los profesores por parte de los directivos institucionales.

En el PCL se destaca el papel de las características de un contexto extranjero, que no facilita el uso del inglés para propósitos comunicativos, y las condiciones socioeconómicas de los padres de familia que no permiten la adquisición de textos y otras ayudas AV a los estudiantes.

En la categoría del PEN se identifican algunas debilidades y características de las políticas educativas nacionales que afectan a los currículos institucionales y que pueden contribuir al bajo nivel de aprendizaje del inglés de los bachilleres. Entre ellos tenemos: las debilidades de la planeación del área en aspectos como los programas analíticos, los objetivos, los contenidos, la metodología y los textos recomendados desde el MEN; la insuficiente inversión en infraestructura física de los colegios del país; la ampliación de la oferta de cursos de capacitación y de actualización profesional, los bajos salarios, y la desvaloración de la profesión docente.

Por último, aunque con menor frecuencia, algunos autores se refieren a la influencia e imposición de políticas socioeconómicas, educativas y tecnológicas de las grandes potencias como Estados Unidos y la Gran Bretaña sobre las políticas sociales, educativas y de enseñanza del inglés en Colombia, que por provenir de dichos países no se adaptan ni son apropiadas para nuestro contexto.

Con relación a los factores internos, los anteriores estudios se refieren a factores inherentes al aprendiz y a los mecanismos internos de aprendizaje de un nuevo idioma. En el primer factor, se resalta el efecto negativo de los siguientes factores: desmotivación, miedo a participar oralmente, bajo autoconcepto sobre sus capacidades para aprender inglés y deficiencias en el manejo del español sobre el aprendizaje efectivo del inglés. En el segundo factor, Bastidas y Muñoz (2018) destacan los insuficientes conocimientos previos y la desatención frecuente de varios estudiantes, mecanismos que afectan el nivel de aprendizaje del inglés. 
Los resultados de las investigaciones en nuestro contexto colombiano nos han permitido identificar una serie de factores que contribuyen a la comprensión del problema macro del bajo nivel de inglés de los bachilleres. Estos factores son más de carácter externo que interno del estudiante. Los estudios que hemos realizado hasta el momento, tanto desde la perspectiva histórica (Bastidas, 2017; Bastidas y Muñoz, 2017; Hidalgo y Caicedo, 2017) y desde la investigación empírica de tipo interpretativo (Bastidas y Muñoz, 2018) nos han proporcionado información que demuestra la complejidad del problema. Por lo tanto, se necesita continuar investigando no solamente para identificar los factores que se relacionan y pueden incidir negativamente, sino también positivamente sobre el aprendizaje del inglés para conformar un marco conceptual que sirva de guía para la comprensión, la explicación y el mejoramiento del nivel de aprendizaje del inglés. En consecuencia, el objetivo general de esta investigación fue analizar los factores externos e internos que tienen una mayor influencia sobre el AILE, según las percepciones de los estudiantes y profesores de bachillerato de Pasto. El anterior objetivo general se desglosó en los siguientes objetivos específicos: 1) identificar el grado de incidencia de factores externos de carácter sociocultural y educativo (externos) sobre el nivel de AILE, según las percepciones de los estudiantes y de los profesores de bachillerato y 2) identificar el grado de incidencia de factores y mecanismos internos de los estudiantes sobre el nivel de AILE, según las percepciones de los estudiantes y de los profesores de bachillerato.

\section{Metodología}

El propósito del macroproyecto, en el que se enmarca este segundo estudio sobre factores externos e internos que tienen una mayor influencia sobre el AILE, pretende lograr un alcance explicativo, sustentado por los niveles previos de la descripción y la correlación. La presente investigación se sustenta en el paradigma empírico-analítico, desde un enfoque cuantitativo para la recolección y el análisis de la información. Además, con el objeto de caracterizar y sustentar los tipos de factores que son percibidos por los profesores y estudiantes como aquellos que inciden en el aprendizaje del inglés en el nivel de educación secundaria, hemos seleccionado el método descriptivo (Escalante Angulo, 1987). Este tipo de paradigma y método nos ha permitido sustentar un marco conceptual que puede tener un poder explicativo, al menos en su etapa inicial.

La información se recolectó mediante la aplicación de dos cuestionarios que incluyen 80 preguntas cada uno, tanto para los estudiantes como para los profesores. La pregunta general para los factores identificados en los ítems fue “ ¿Qué tanto influye el siguiente factor para obtener un buen nivel de aprendizaje del inglés en el bachillerato? Los participantes tenían que colocar una $\mathrm{x}$ debajo de las casillas que se ubican en una escala de 1 a 5, donde 1 representa muy poca y 5 muchísima contribución del factor indicado para que los estudiantes obtengan un buen nivel de AILE. Estos instrumentos se construyeron con base en los factores que han sido identificados en la literatura, tanto del campo de AsL como del ALE. Éstos se clasificaron en factores relacionados con el Contexto Sociocultural y Educativo (externos) $y$ en factores inherentes al Aprendiz y mecanismos de Aprendizaje de una Lengua Extranjera (internos). Esta última denominación se la adaptó de los escritos de Ellis (1994). Los primeros, a su vez, se subclasificaron en: Ambiente de Aprendizaje en el Aula (AAA), Currículo Institucional (CI), Papel de las Políticas Educativas Nacionales (PEN), Papel de la Comunicad Local (PCL), Preparación Profesional de los Profesores (PPP) y Papel de la Comunidad Internacional (PCL). Los segundos se agruparon en cognitivos, lingüísticos, afectivos e internos de carácter sociocultural. Los dos cuestionarios se aplicaron a un grupo de 113 estudiantes recién egresados del bachillerato y a 25 profesores. Con el objeto de revisar las preguntas en cuanto a su claridad, precisión y especificidad y para lograr una mayor confiabilidad y validez del cuestionario, éste se aplicó a una muestra de 40 estudiantes recién egresados de bachillerato. 


\section{Resultados}

A continuación, presentamos los resultados del análisis de los cuestionarios aplicados a los estudiantes y a los profesores con el objetivo de analizar los factores externos e internos, que contribuyen favorablemente a obtener un buen nivel de AILE.

\section{Contexto sociocultural y educativo}

El contexto sociocultural y educativo se refiere al ambiente físico y psicológico en el que se lleva a cabo el proceso de AILE, mediado por las acciones e interacciones, tanto de los estudiantes, profesores, directivos y padres de familia, como de los recursos audiovisuales y las actividades que se desarrollan en clase y en las instituciones. Además, el contexto incluye otros agentes como son las autoridades educativas, las políticas y lineamientos educativos, la infraestructura y los recursos financieros, los medios de comunicación, incluidas las TIC entre otros, tanto a nivel local, como nacional e internacional (Bastidas y Muñoz, 2018). Los resultados correspondientes al primer objetivo específico que pretende "identificar el grado de incidencia de factores externos de carácter sociocultural y educativo sobre el nivel de AILE, según las percepciones de los estudiantes y de los profesores de bachillerato" se muestran a continuación.

En la figura 1 se presentan los resultados, tanto de profesores como de estudiantes sobre los factores externos que hacen parte del tema denominado contexto sociocultural y educativo, que según ellos pueden contribuir positivamente en el nivel de AILE. El reporte se hace con base en las medias aritméticas obtenidas en cada una de las categorías, las cuales se obtuvieron en una escala de 1 a 5 , donde 1 nos indica muy poca contribución y 5 muchísima contribución. Para propósitos de la interpretación de los resultados establecimos con anticipación que una contribución equivalente al 70\% (3.5 en la escala de 1 a 5) se la puede considerar como una categoría que contribuye favorablemente al aprendizaje.

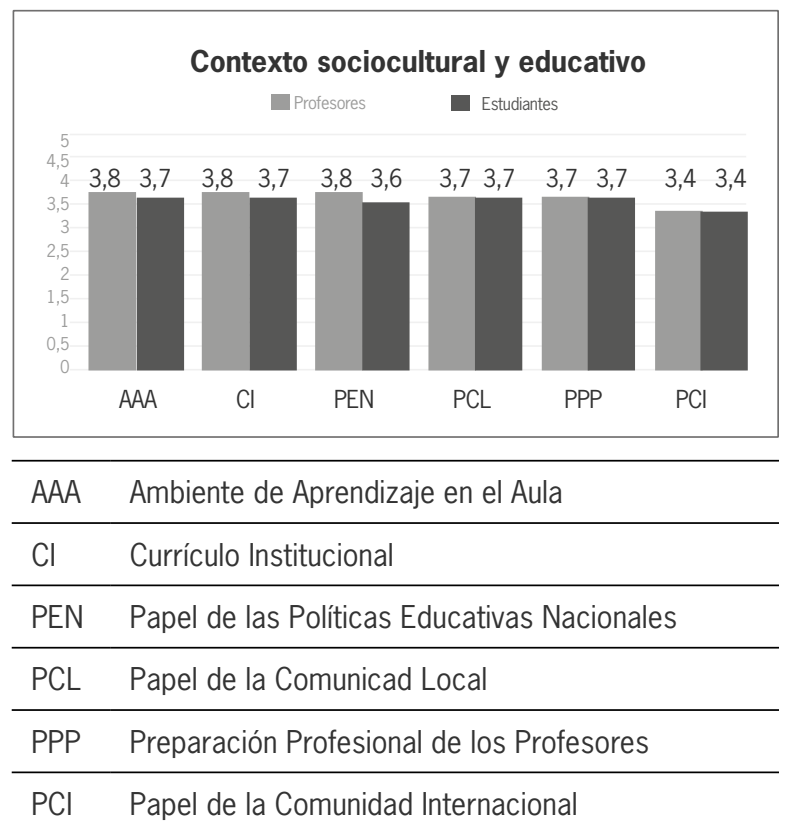

Figura 1. Categorías del contexto sociocultural y educativo que inciden favorablemente en el nivel de AlLE.

Aunque con mínimas diferencias, tanto profesores como estudiantes identificaron factores relacionados con cinco tipos de categorías socioculturales y educativas que contribuyen favorablemente al AILE: Ambiente de Aprendizaje en el Aula (AAA), Currículo Institucional (CI), Papel de la Comunidad Local (PCL) Papel de las Políticas Educativas Nacionales (PEN) y Preparación Profesional de los Profesores (PPP).

El análisis más detallado de este primer resultado indica: primero, que tanto profesores como estudiantes coinciden plenamente en el grado de influencia de los factores PCL, PPP y PCI; segundo, hay coincidencia, con una pequeña diferencia, entre las percepciones de profesores y estudiantes en las categorías de: AAA (profesores: media 3.8 y estudiantes: media 3.7), CI (profesores media 3.8 y estudiantes: media 3.7) y PEN (profesores media 3.8 y estudiantes media 3.6) y tercero, la gráfica nos indica que los profesores consideran que las categorías de AAA, CI y PEN son las que más contribuyen, mientras que para los estudiantes las categorías de mayor contribución al aprendizaje son AAA, CI y PPP. Finalmente, el factor sobre Papel de la Comunidad Internacional (PCI) contribuye en menor escala 
(3.4) sobre el nivel de AILE, según los profesores y estudiantes.

\section{Factores inherentes al aprendiz y mecanismos de aprendizaje de una lengua extranjera}

En el proceso de ASL y de ALE intervienen mecanismos y factores intrapersonales de tipo biológico, cognitivo, afectivo y lingüístico, mediados por factores interpersonales de carácter sociocultural. El análisis de la información de tipo cualitativo, recogida por Bastidas y Muñoz (2018) inicialmente permitió identificar dos categorías que se han denominado factores inherentes al aprendiz $y$ mecanismos internos de aprendizaje, denominación tomada de los escritos de Ellis (1994).

En la figura 2 se presentan los resultados que han permitido dar respuesta al segundo objetivo específico de la investigación: "Identificar el grado de incidencia de factores y mecanismos internos de los estudiantes sobre el nivel de AILE, según las percepciones de los estudiantes y de los profesores de bachillerato".

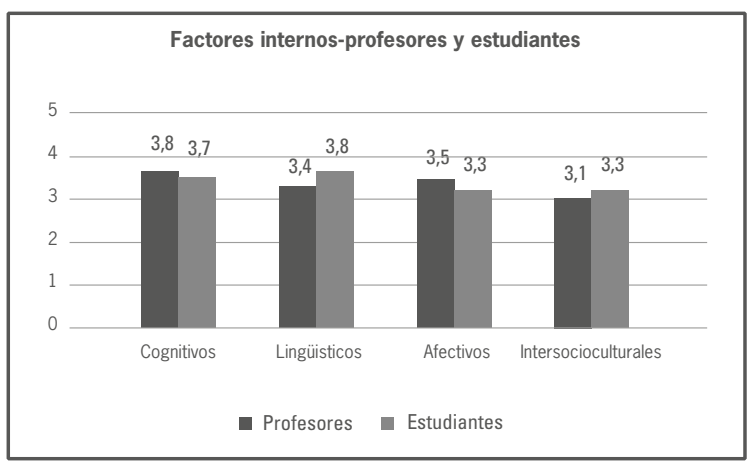

Figura 2. Factores internos y mecanismos de aprendizaje que inciden favorablemente en el nivel de AllE.

Los resultados de la figura anterior muestran que mientras para los profesores los factores internos que contribuyen al rendimiento académico en inglés son los de tipo cognitivo (3.8) y afectivo (3.6), para los estudiantes son los de tipo lingüístico (3.8) y cognitivo (3.7). Como se puede observar, no hay coincidencias absolutas entre las percepciones de los estudiantes y de los profesores sobre el grado de incidencia de los factores internos. Además, mientras para los profesores los factores de tipo afectivo contribuyen favorablemente (3.6), para los estudiantes éstos contribuyen en menor escala (3.3). Finalmente, tanto para profesores (3.1) como para estudiantes (3.3), los factores de tipo interpersonalsociocultural contribuyen con menor intensidad sobre el nivel de AILE.

\section{Factores que tienen una mayor incidencia en el nivel de AlLE de los bachilleres}

En la figura 3 se presenta una síntesis de los resultados obtenidos para el logro de los objetivos específicos arriba indicados y que nos permiten visualizar el alcance del objetivo general de esta investigación que se expresó así: "analizar los factores internos y externos que tienen una mayor influencia sobre el nivel de AILE, según las percepciones de los estudiantes y profesores de bachillerato de Pasto, Colombia".

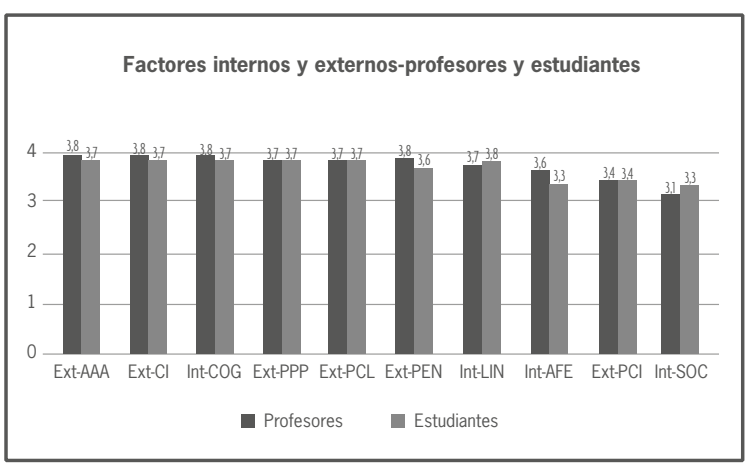

Figura 3. Síntesis de factores externos e internos que inciden favorablemente en el nivel de AlLE del bachillerato.

Los resultados anteriores indican que los factores externos tienden a ser percibidos como de mayor incidencia en el rendimiento académico, tanto por los profesores como por los estudiantes. Esto se demuestra por la obtención de mayores puntajes (en promedio), de 5 categorías de factores externos: AAA, CI, PEN, PCL y PPP, comparados con 3 categorías de factores internos: Cognitivos, Lingüísticos y Afectivos. Los resultados anteriores concuerdan con la tendencia en el campo de ALE a resaltar una mayor incidencia de los factores externos que de los internos sobre el aprendizaje del inglés (Bastidas y Muñoz, 2018; Brown, 2007). Sin embargo, es importante señalar que los factores cognitivos fueron percibidos 
con casi igual grado de incidencia que los factores externos de AAA y CI (media 3.8 y 3.7); además, para los estudiantes los factores lingüísticos también obtuvieron un similar grado de incidencia (3.8) al de los anteriores factores externos.

\section{Discusión}

Como se indicó anteriormente, en esta investigación se utilizaron las categorías encontradas en el primer estudio de tipo interpretativo-cualitativo (Bastidas y Muñoz, 2018), las cuales se relacionan con los temas de Contexto Sociocultural y Educativo, a saber: Ambiente de Aprendizaje en el Aula (AAA), Currículo Institucional (CI), Papel de las Políticas Educativas Nacionales (pen), Papel de la Comunicad Local (PCL), Preparación Profesional de los Profesores (PPP) y Papel de la Comunidad Internacional (PCI) y con el tema sobre factores Inherentes al Aprendiz y Mecanismos de Aprendizaje de una Lengua Extranjera, los que fueron agrupados en Cognitivos,
Lingüísticos, Afectivos e internos de carácter Sociocultural. Además, se tuvieron en cuenta los factores identificados en la revisión de la literatura, tanto en los campos de ASL como de ALE. Con el objeto de corroborar o refutar los hallazgos del anterior estudio basado en un enfoque cualitativo, en la sección anterior reportamos los resultados de este estudio discriminados por categorías, con base en un enfoque cuantitativo. En esta sección se analizan los factores externos e internos que conformaron cada una de las categorías y que fueron percibidos por los estudiantes y profesores como factores que tienen un mayor o menor grado de incidencia sobre el aprendizaje. El análisis y la discusión, también, se realizan en forma integrada de los dos tipos de categorías de factores para sustentar el objetivo general del estudio.

En las dos tablas siguientes se muestran los factores ordenados, tanto internos (resaltados en color) como externos, identificados por los estudiantes y por los profesores.

Tabla 1. Factores externos e internos percibidos por los estudiantes ordenados de mayor a menor grado de incidencia sobre el nivel de AlLE, según las medias aritméticas obtenidas.

\begin{tabular}{|c|c|c|c|c|}
\hline No. & Cód. & Categorías & Media & $\begin{array}{l}\text { Desv. } \\
\text { Stnd }\end{array}$ \\
\hline 1 & PPP & Preparación profesores: Buen nivel conocimientos del profesor. & 4.4 & 0.7 \\
\hline 2 & ISOC & Inter-Socioculturales: Actitud positiva aprendizaje. & 4.4 & 0.6 \\
\hline 3 & PEN & $\begin{array}{l}\text { Papel Políticas Educativas Nacionales: Garantizar } \\
\text { financiación a la educación pública. }\end{array}$ & 4.3 & 1.0 \\
\hline 4 & ISOC & Inter-Socioculturales: Estudiar inglés desde edad temprana. & 4.3 & 0.9 \\
\hline 5 & PPP & Preparación profesores: Actitud positiva del profesor hacia la enseñanza. & 4.2 & 0.8 \\
\hline 6 & PPP & Preparación profesores: Alta motivación del profesor para enseñar inglés. & 4.2 & 0.8 \\
\hline 7 & $\mathrm{COA}$ & Cognitivos: Prestar atención a explicaciones y actividades de clase. & 4.2 & 0.6 \\
\hline 8 & PPP & Preparación profesores: Diversas estrategias aprendizaje aplicadas por el profesor. & 4.1 & 1 \\
\hline 9 & PPP & Preparación profesores: Conocimiento diferencias español e inglés del profesor. & 4.1 & 0.8 \\
\hline 10 & PPP & $\begin{array}{l}\text { Preparación profesores: Conocimiento y habilidades } \\
\text { del profesor para enseñar inglés. }\end{array}$ & 4.1 & 0.8 \\
\hline 11 & PPP & Preparación profesores: Alta preparación del profesor para cada clase. & 4.1 & 0.7 \\
\hline 12 & PPP & Preparación profesores: Alta autoestima del profesor. & 4.0 & 0.7 \\
\hline 13 & $\mathrm{PCl}$ & $\begin{array}{l}\text { Papel comunidad internacional: Conocimiento y aplicación tendencias } \\
\text { internacionales en enseñanza del inglés. Importancia del inglés a nivel mundial. }\end{array}$ & 4.0 & 0.6 \\
\hline
\end{tabular}


Universidad Pedagógica Nacional

Facultad de Humanidades

\begin{tabular}{|c|c|c|c|c|}
\hline No. & Cód. & Categorías & Media & $\begin{array}{l}\text { Desv. } \\
\text { Stnd }\end{array}$ \\
\hline 14 & AAA & Ambien Aprendizaje en Aula: Realizar actividades variadas y trabajo cooperativo. & 3.9 & 0.8 \\
\hline 15 & PCL & $\begin{array}{l}\text { Papel Comunidad Local: Estudiar inglés por propia } \\
\text { iniciativa. Hacer tareas fuera de aula. }\end{array}$ & 3.9 & 0.8 \\
\hline 16 & $\mathrm{Cl}$ & Papel Comunidad Internacional: Utilizar textos de EEUU. Existencia de ayudas AV. & 3.9 & 0.7 \\
\hline 17 & $\mathrm{Cl}$ & $\begin{array}{l}\text { Currículo Institucional: Coordinación y seguimiento } \\
\text { de programas de un grado a otro. }\end{array}$ & 3.9 & 0.8 \\
\hline 18 & COM & $\begin{array}{l}\text { Motivación: Interés, importancia, utilidad, preferencia; } \\
\text { auto-concepto positivo capacidades. }\end{array}$ & 3.9 & 0.6 \\
\hline 19 & LIN & Lingüísticos: Entender diferencias entre español e inglés. & 3.9 & 0.8 \\
\hline 20 & AAA & Ambiente Aprendizaje en el Aula: Uso apropiado de texto. Ayudas AV. e internet. & 3.8 & 0.9 \\
\hline 21 & AAA & Ambiente Aprendizaje en el Aula: Demostrar buen comportamiento. & 3.8 & 0.8 \\
\hline 22 & AAA & Ambiente Aprendizaje en el Aula: Ser evaluados semanalmente de diversas formas. & 3.7 & 0.8 \\
\hline 23 & PEN & $\begin{array}{l}\text { Papel Políticas Educativas Nacionales: Establecer objetivos } \\
\text { alcanzables, emitir programas y lineamientos curriculares. }\end{array}$ & 3.7 & 0.8 \\
\hline 24 & PPP & Preparación profesores: Conocimiento de estilos de aprendizaje de estudiantes. & 3.7 & 0.9 \\
\hline 25 & PPP & $\begin{array}{l}\text { Preparación profesores: Conocimiento y sensibilidad realidad } \\
\text { económica social y cultural de los jóvenes. }\end{array}$ & 3.7 & 0.9 \\
\hline 26 & $\mathrm{COC}$ & Cognitivos: Conciencia capacidades otros compañeros aprender inglés. & 3.7 & 0.8 \\
\hline 27 & LIN & Lingüísticos: Habilidad comunicación oral y escrita en español. & 3.7 & 0.7 \\
\hline 28 & ISOC & Inter-Socioculturales: Actitud positiva cultura americana. Valorar constumbre. & 3.7 & 0.7 \\
\hline 29 & AAU & Afectivos: Alta autoestima. & 3.7 & 0.9 \\
\hline
\end{tabular}

En la tabla 1 se presentan los factores internos y externos, reconocidos por los estudiantes y ordenados de mayor a menor, según la media de los puntajes de las respuestas a cada una de las preguntas del cuestionario. Un hallazgo importante de esta tabla es que en el proceso de AILE no se puede afirmar de manera contundente, que son todos los factores externos o internos los que tienen mayor incidencia, ya sea positiva o negativa. Por el contrario, tal como lo afirman Mahmoudi y Mahmoudi (2015), la incidencia depende de la fuerza con que los participantes perciben que un determinado factor interno o externo, contribuye al proceso de aprendizaje, concepto que depende de su conocimiento o experiencia como aprendices. En la tabla anterior, tanto un factor externo: buen nivel de conocimientos del profesor, como un factor interno: actitud positiva hacia el aprendizaje del inglés de los estudiantes son los de mayor contribución (4.4). El hallazgo de los dos últimos factores es importante para el sustento de aquellos factores internos que parecen tener una mayor incidencia en el nivel de aprendizaje del inglés en un contexto extranjero.

Luego se ubica otro factor externo: garantizar financiación a la educación pública y dos factores internos: estudiar inglés desde la edad temprana (4.3) y prestar atención en clase (4.2). Estos resultados también son novedosos, ya que indican que los estudiantes son conscientes del efecto de la financiación de la educación, como un factor de las políticas nacionales y de la necesidad de estudiar inglés desde temprana edad, con el objeto de obtener un mejor 
nivel en su aprendizaje. Sin embargo, no se puede desconocer que la tendencia en los resultados de la tabla 1 es a identificar un mayor número de factores externos, que contribuyen al aprendizaje del inglés, en comparación con el efecto de los factores internos. Además, nuevamente se destaca la presencia de factores relacionados con la PPP en los lugares más altos de la tabla. Esto significa que, para los estudiantes, tanto las características personales como la preparación profesional de los profesores hacen parte de aquellos factores que pueden contribuir positiva o negativamente al aprendizaje del inglés, aspecto que ha sido resaltado en la literatura de nuestro país (Bastidas y Muñoz, 2018; Cardona y Hanley, 1985; y Navarrete, 1970).

En la tabla 2 se muestran las categorías y factores externos e internos ordenados según la media aritmética de las respuestas de los profesores. Tal como se indicó en la tabla anterior, los factores internos se resaltan en color.

Tabla 2. Factores externos e internos percibidos por los profesores ordenados de mayor a menor grado de incidencia sobre el nivel de aprendizaje del inglés, según las medias aritméticas obtenidas.

\begin{tabular}{|c|c|c|c|c|}
\hline No. & Cód. & Categorías & Media & $\begin{array}{l}\text { Desv. } \\
\text { Stnd }\end{array}$ \\
\hline 1 & PPP & Preparación profesores: Actitud positiva del profesor hacia la enseñanza. & 4.6 & 0.5 \\
\hline 2 & PPP & $\begin{array}{l}\text { Preparación profesores: Diversidad estrategias } \\
\text { que aplique el profesor para enseñar. }\end{array}$ & 4.4 & 0.7 \\
\hline 3 & PPP & Preparación profesores: Buen nivel conocimientos del profesor. & 4.4 & 0.7 \\
\hline 4 & PPPP & Preparación profesores: Alta motivación del profesor para enseñar inglés & 4.3 & 0.6 \\
\hline 5 & PEN & $\begin{array}{l}\text { Papel Políticas Educativas Nacionales: Garantizar } \\
\text { financiación a la educación pública. }\end{array}$ & 4.3 & 1.1 \\
\hline 6 & $\mathrm{COA}$ & Cognitivos: Prestar atención a explicaciones y actividades de clase. & 4.3 & 0.7 \\
\hline 7 & $\mathrm{Cl}$ & Currículo Institucional: Tener un número menor de estudiantes por curso. & 4.2 & 1.2 \\
\hline 8 & PPP & $\begin{array}{l}\text { Preparación Profesores: Conocimiento y habilidades } \\
\text { del profesor para enseñar inglés. }\end{array}$ & 4.2 & 0.6 \\
\hline 9 & PPP & Preparación Profesores: Alta preparación del profesor para cada clase. & 4.2 & 0.8 \\
\hline 10 & AAA & Ambiente Aprendizaje en el Aula: Ambiente físico apropiado. & 4.1 & 1.0 \\
\hline 11 & PPP & Preparación Profesores: Conocimiento de estilos de aprendizaje de estudiantes. & 4.1 & 0.6 \\
\hline 12 & PPP & Preparación Profesores: Alta autoestima del profesor. & 4.1 & 0.5 \\
\hline 13 & PPP & Preparación Profesores: Comprensión estado afectivo de los estudiantes. & 4.1 & 0.5 \\
\hline 14 & AAA & Ambiente Aprendizaje en el Aula: Realizar actividades variadas y trabajo cooperativo. & 4.1 & 0.5 \\
\hline 15 & COM & $\begin{array}{l}\text { Motivación: Interés, importancia, utilidad, preferencia, } \\
\text { auto-concepto positivo capacidades. }\end{array}$ & 4.1 & 0.6 \\
\hline 16 & PCL & $\begin{array}{l}\text { Papel Comunidad Local: Estudiar inglés por propia } \\
\text { iniciativa, hacer tareas fuera de aula. }\end{array}$ & 4.0 & 0.7 \\
\hline
\end{tabular}


Universidad Pedagógica Nacional

Facultad de Humanidades

\begin{tabular}{|c|c|c|c|c|}
\hline No. & Cód. & Categorías & Media & $\begin{array}{l}\text { Desv. } \\
\text { Stnd }\end{array}$ \\
\hline 17 & PPP & $\begin{array}{l}\text { Preparación profesores: Conocimiento y sensibilidad realidad } \\
\text { económica, social y cultural de los jóvenes. }\end{array}$ & 4.0 & 0.6 \\
\hline 18 & AAA & Ambiente Aprendizaje en el Aula: Uso apropiado de texto, ayudas AV. e internet. & 3.9 & 0.8 \\
\hline 19 & $\mathrm{Cl}$ & $\begin{array}{l}\text { Currículo institucional: Mayor intensidad horaria semanal, } \\
\text { ubicación clases de inglés en el horario. }\end{array}$ & 3.9 & 0.9 \\
\hline 20 & $\mathrm{PCl}$ & $\begin{array}{l}\text { Papel comunidad internacional: Conocimiento y aplicación tendencias } \\
\text { internacionales en enseñanza del inglés. Importancia del inglés a nivel mundial. }\end{array}$ & 3.9 & 0.8 \\
\hline 21 & AAA & Ambiente Aprendizaje en el Aula: Demostrar buen comportamiento. & 3.9 & 0.6 \\
\hline 22 & CEA & Cognitivos: utilizar medios visuales, auditivos y kinestésicos para aprender. & 3.9 & 0.6 \\
\hline 23 & PEN & $\begin{array}{l}\text { Papel Políticas Educativas Nacionales: } \\
\text { Medición adecuada a través de pruebas SABER 1.1. }\end{array}$ & 3.8 & 0.8 \\
\hline 24 & $\mathrm{Cl}$ & $\begin{array}{l}\text { Currículo Institucional: Coordinación y seguimiento } \\
\text { de programas de un grado a otro. }\end{array}$ & 3.8 & 1.0 \\
\hline 25 & PEN & $\begin{array}{l}\text { Papel Políticas Educativas Nacionales: Establecer objetivos } \\
\text { alcanzables, emitir programas y delineamientos curriculares. }\end{array}$ & 3.8 & 0.8 \\
\hline 26 & PPP & Preparación profesores: Conocimiento diferencias español e inglés del profesor. & 3.8 & 0.8 \\
\hline 27 & CES & Cognitivos: Utilizar estrategias, conciencia aprendizaje auto-recompensa. & 3.8 & 0.6 \\
\hline 28 & PPP & Preparación profesores: Bajo nivel de ansiedad del profesor para enseñar & 3.7 & 1.0 \\
\hline 29 & AAU & Afectivos: Alta autoestima & 3.7 & 0.8 \\
\hline 30 & $\mathrm{CCP}$ & Cognitivos: Conocimientos previos. & 3.7 & 0.7 \\
\hline
\end{tabular}

Los resultados de la tabla 2, aunque no muestran el mismo patrón de organización de los factores externos e internos según los estudiantes, sí demuestran que tanto factores externos como algunos internos se entremezclan con base en el grado de contribución que reconocen los profesores de bachillerato, según su conocimiento y experiencia como aprendices y enseñantes (Mahmoudi y Mahmoudi, 2015). Por otra parte, los profesores resaltan 4 factores correspondientes a la categoría de Ppp, tales como: mostrar actitud positiva del profesor hacia la enseñanza (4.6), aplicar diversas estrategias para enseñar (4.4), tener un buen nivel de conocimientos (4.4) y demostrar alta motivación para enseñar inglés (4.3). Este resultado corrobora lo encon- trado en los datos de los estudiantes, y significa que las características personales y la preparación profesional de los profesores son factores esenciales que contribuyen al aprendizaje del inglés, según la percepción de los profesores (Bastidas \& Muñoz, 2018; Cardona \& Hanley, 1985; Navarrete, 1970).

En la tabla 2, se puede observar, también, que los profesores incluyen un factor externo sobre el PEN: garantizar financiación a la educación pública (4.3) en el quinto lugar. Esta tendencia coincide con la percepción de los estudiantes, quienes, a pesar de haberlo ubicado en el tercer lugar, la magnitud de su contribución para el aprendizaje (4.3) es equivalente al promedio otorgado por los profesores. Esto constituye un llamado a las autoridades educativas 
institucionales, locales y nacionales, así como también a las gubernamentales para que se asigne una mayor financiación a la educación en general.

Con relación a los factores internos, los resultados muestran que los profesores resaltan dos: prestar atención a explicaciones y actividades de clase (4.3) y demostrar interés, importancia, utilidad, preferencia, autoconcepto positivo (4.1), dimensiones correspondientes a la motivación para el aprendizaje, tal como lo ha establecido la literatura en el campo de la motivación hacia el aprendizaje en general (ej.: Eccles, 2005; Schunk y Pintrich, 2008; Wigfield, 1994; Wigfield, Tonks, y Eccles, 2004) y en el área de la motivación hacia el aprendizaje del inglés (ej.: Alsayed, 2003; Cheng y Dörnyei, 2007; Dörnyei, 1994; Gardner, 1985). Estos resultados coinciden con la identificación realizada por los estudiantes, quienes también los ubican entre los primeros 18 factores de mayor contribución al aprendizaje. Además de corroborar el papel esencial que parece jugar la motivación en el aprendizaje del inglés, estos resultados identifican un hallazgo importante, que contribuye a la literatura en el campo del aprendizaje del inglés, que es la identificación de la atención como un mecanismo relevante para asegurar un mayor aprendizaje del inglés (Bastidas y Muñoz, 2018; Sanz, 2005).

El desglose de las categorías en sus correspondientes factores ha proporcionado dos contribuciones sobresalientes para los hallazgos del estudio. Por una parte, se han reconocido y sustentado factores que han sido identificados en la literatura de los campos de Ale y de ASL. Por otra parte, se han resaltado otros factores que contribuyen al aprendizaje del inglés. Mientras los profesores identificaron 15 factores externos y 2 internos, los estudiantes reconocieron 10 factores externos y 3 internos. Todos los anteriores factores sobrepasaron la media aritmética de 4.0, correspondiente a una contribución en alto grado. En consecuencia, se proporcionan evidencias desde el paradigma empírico-analítico, con un enfoque cuantitativo, que permiten sustentar y revisar la hipótesis formulada por Bastidas y Muñoz (2018), desde un paradigma interpretativo-cualitativo y que ahora se expresa así: "En un contexto de lengua extranjera, el nivel de inglés de los bachilleres se relaciona con un mayor número de factores socioculturales y educativos externos que con mecanismos y factores internos del estudiante".

Finalmente, todos los datos anteriores nos demuestran que el problema del proceso de AILE es un problema complejo que incluye una cantidad de categorías de factores, tanto de carácter externo como interno, que muy seguramente actúan e interactúan de manera simultánea, dinámica, incierta e impredecible; tal como afirma Morin (1994) sucede en el comportamiento de los fenómenos sociales y naturales. Por lo tanto, los actores principales del proceso de aprendizaje: estudiantes y profesores, autoridades educativas institucionales, locales y nacionales, así como también los gobernantes deben tenerlos en cuenta y enfocar las políticas, la toma de decisiones y las acciones para contribuir al mejoramiento efectivo del nivel de inglés de los bachilleres de la ciudad de Pasto, en particular y de los bachilleres del país en general.

Con el objeto de ayudar a entender la complejidad del proceso de AILE, en la Figura 4 se presenta un modelo conceptual. Este modelo se ha construido con base en la identificación de 18 factores internos y 37 externos que sirvieron de base para la construcción de los dos cuestionarios diligenciados por los profesores y los estudiantes.

Tal como se reportó en el primer estudio (Bastidas y Muñoz, 2018) y ahora en el presente, los anteriores factores se agruparon en temas y categorías para facilitar el análisis y reporte de los resultados. Los dos grandes temas se denominaron "Contexto sociocultural y educativo" y "Factores inherentes al aprendiz y mecanismos de aprendizaje de una lengua extranjera”. En los dos estudios se logró identificar la presencia de factores que se relacionan con las categorías: AAA, CI, PEN, PCL, PPP y PCI que corresponden al tema de Contexto Sociocultural y las categorías derivadas del tema denominado Factores inherentes al aprendiz y Mecanismos de aprendizaje de una lengua extranjera, tales como 
Factores Cognitivos, Lingüísticos, Afectivos e Interpersonales de carácter sociocultural.

En la siguiente figura se presenta el modelo que contiene algunas modificaciones de los términos utilizados para denominar los temas, las categorías y los factores, de tal manera que se aproximen a la terminología estándar de los campos de ASL y de ALE.

Figura 4. Marco conceptual del proceso de AlLE, mediado por factores socio-culturales e internos.

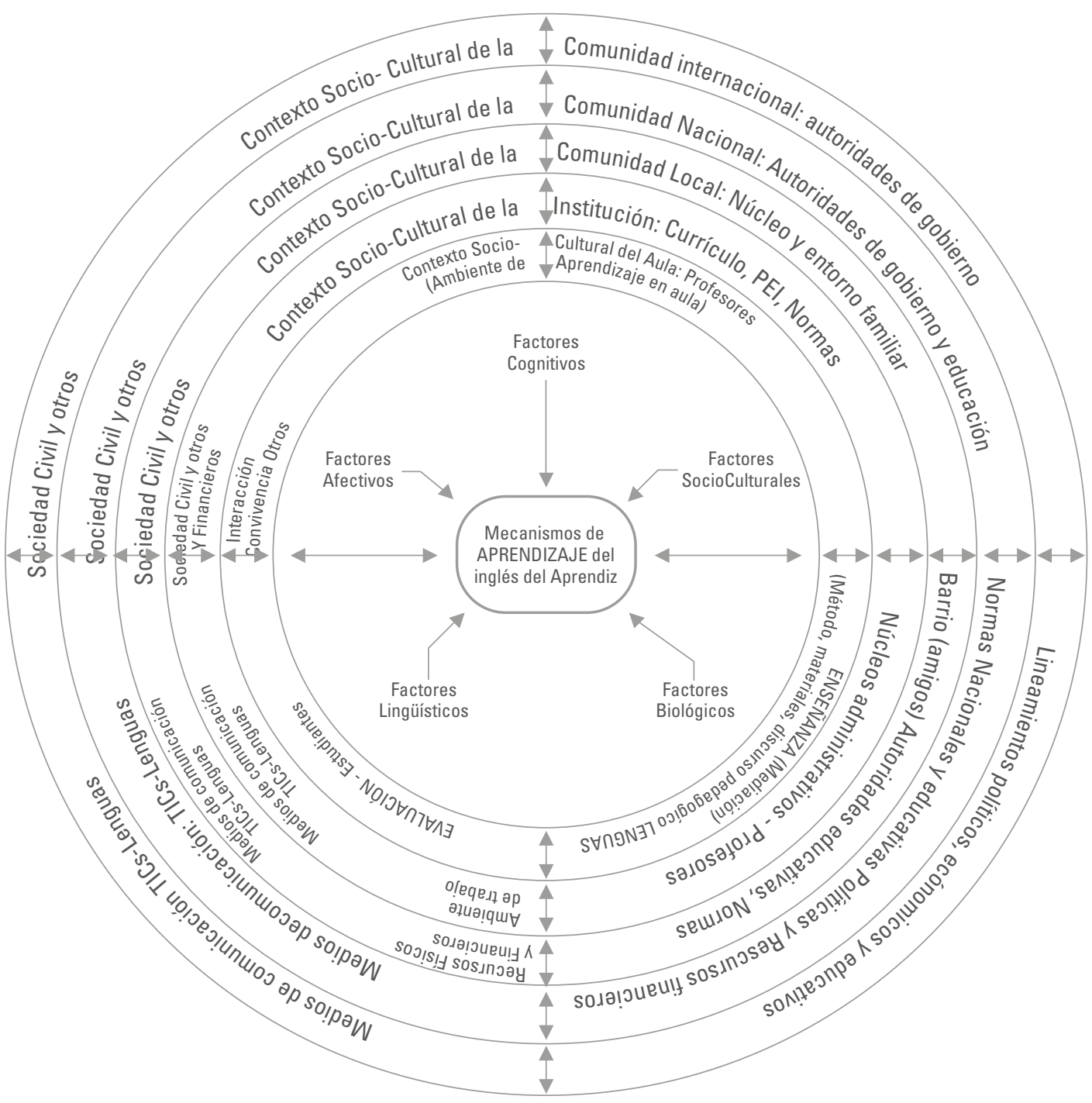


Este modelo se caracteriza por reflejar la complejidad, la multidimensionalidad, la interacción sociocultural, la dinámica, el orden y el desorden, la incertidumbre, etc., (Morin, 1994) del proceso de aprendizaje del inglés en un contexto de lengua extranjera. Las flechas perpendiculares representan las posibles influencias e interacciones sobre el aprendizaje y las flechas circulares muestran la dinámica de los agentes y de las actividades que se desarrollan en cada uno de los tipos de contexto sociocultural que sirve de entorno favorable o desfavorable para el AILE. En el modelo se incluye un factor que, si bien no se destacó en la discusión, si fue mencionado por los profesores y estudiantes: los medios de comunicación, incluidas las Tic y la presencia no sólo de material audiovisual en español, sino también en otros idiomas. Se espera que estas herramientas jueguen un papel más preponderante como motivador y facilitador del AILE en el futuro.

Como todo modelo conceptual, éste no incluye todos los factores y sub-factores que están presentes en cada categoría, ni tampoco refleja la dinámica de los procesos y de los contextos, así como tampoco representa los procesos internos de los actores de cada contexto. Sin embargo, el modelo sirve para identificar los temas, categorías y factores sobresalientes que actúan en el proceso de AILE y que permiten formular hipótesis y teorías que faciliten la descripción, la comprensión y la explicación de este proceso en investigaciones futuras.

Finalmente, es importante resaltar que este modelo sirve para revisar algunos aspectos del modelo planteado deductivamente por Bastidas en 2016, cuyo objetivo fue construirlo y compararlo con los modelos de enseñanza y aprendizaje de una L2 propuestos por autores como Mackey (1970), Stern (1983) y Strevens (1977), para sustentar una nueva propuesta de programa de educación en lenguas extranjeras con base en lineamientos de tipo curricular. La comparación anterior, además, sirvió para demostrar que el proceso de ALE es mucho más complejo que el proceso de ASL, dada la existencia de varios factores adicionales que no se presentan en el ASL; para señalar que las diferencias entre la enseñanza y el aprendizaje del ISL y del ILE sobrepasan considerablemente la diferencia tradi- cional centrada en la presencia o ausencia del uso de la L2 en el contexto (ej.: Muñoz, 2002; Richards, Platt y Weber, 1985) y para destacar la necesidad de sustentar al proceso de ALE desde una perspectiva socio-cultural e histórica (Johnson, 2009; Vygotsky, 1978), o socio-cognitiva (Batstone, 2010; Seedhouse, 2010), para lo cual se necesita continuar desarrollando muchos más estudios de este y otros tipos, tanto a nivel local, como nacional e internacional.

\section{Conclusiones, implicaciones e impacto de la investigación}

La complejidad del problema de AILE, tanto a nivel internacional como nacional y local, justifica la continuidad de las investigaciones, que no solamente contribuyan a comprender el problema desde el paradigma interpretativo, sino también a explicarlo desde el paradigma empírico-analítico; además, a contribuir a su mejoramiento desde el paradigma sociocrítico. Esta investigación, de carácter empírico-analítico ha proporcionado unos resultados que nos han permitido, por una parte, corroborar la existencia de factores externos e internos que según la literatura nacional e internacional inciden en el nivel de AILE. Por otra parte, resaltar otros factores que influyen en dicho aprendizaje según la percepción de los profesores y estudiantes egresados de la educación secundaria y que no han sido resaltados en la literatura.

Los resultados de los objetivos específicos de la investigación indican, en primer lugar, que las categorías de factores de carácter sociocultural y educativo, tales como: Ambiente de aprendizaje en el Aula, Currículo institucional, Preparación Profesional del Profesor, Papel de la Comunidad Local y Papel de las Políticas Educativas Nacionales tienden a ser percibidas como de mayor influencia sobre el nivel de aprendizaje del inglés, tanto por profesores como por estudiantes, que los factores inherentes al aprendiz y los mecanismos internos de aprendizaje. En segundo lugar, los factores internos de mayor influencia son los de carácter cognitivo y lingüístico. Sin embargo, al desglosar las anteriores categorías en sus factores específicos 
se pudo detectar que hay factores internos, como la actitud, la atención y la motivación de los estudiantes, que son percibidos de mucha influencia a la par con otros factores externos socioculturales. Asimismo, se resaltan varios factores relacionados con la Preparación Profesional del Profesor. Es decir, si bien hay una tendencia a resaltar las categorías de factores externos de tipo sociocultural y educativo como de mayor influencia, no se debe desconocer que existen determinados factores internos inherentes al aprendiz que son percibidos por profesores $y$ estudiantes como de gran influencia en el aprendizaje del inglés, tal como lo han propuesto autores como Mahmoudi y Mahmoudi (2015).

La importancia de esta investigación se sustenta en las implicaciones y el impacto que pueden tener sus resultados en los campos de la Lingüística Aplicada y del Aprendizaje del Inglés como Lengua Extranjera. Las contribuciones las clasificamos en teóricas, prácticas y administrativas. En el aspecto teórico, la identificación de las categorías sobre los factores externos e internos discutidos en el anterior estudio (Bastidas y Muñoz, 2018), desde un paradigma interpretativo y en el presente, desde un paradigma empírico-analítico contribuyen a la revisión y sustento de modelos teóricos, tales como los presentados por Bastidas (2016), el cual intenta representar la complejidad del proceso de aprendizaje del inglés en un contexto extranjero y además, contribuir a la explicación del problema del bajo nivel de aprendizaje del inglés de los egresados de la educación secundaria, no solamente a nivel nacional sino también internacional. Una vez identificados los factores proponemos un marco conceptual que incluye no solamente los factores, sino que además sugiere relaciones entre ellos, elemento fundamental para la comprensión y explicación del fenómeno de estudio. El anterior marco conceptual puede tener un impacto en los campos arriba indicados, ya que proporciona unos hallazgos iniciales provenientes de los propios actores del aprendizaje del inglés, como lo son los estudiantes de bachillerato y de la enseñanza de dicho idioma, como lo son los profesores, quienes si bien representan a un caso específico de un contexto de aprendizaje en un contexto extranjero, seguramente servirán de motivación a otros investigadores nacionales e internacionales a replicar este tipo de estudios con el objeto de sustentar el marco conceptual inicial, formular nuevas hipótesis y contribuir a la sustentación de una o más teorías que expliquen el fenómeno del bajo nivel de aprendizaje del inglés como lengua extranjera.

En lo práctico, se continúa proporcionando información valiosa sobre los factores, tanto externos como internos que parecen contribuir favorable o desfavorablemente al aprendizaje del inglés como lengua extranjera en la escuela secundaria para que, tanto los directivos institucionales, como los profesores, estudiantes y padres de familia tengan una mayor comprensión del problema. Los resultados de ésta y otras investigaciones indicadas en la introducción constituyen un llamado a los actores anteriores, para que tengan en cuenta cada uno de los anteriores factores identificados para proponer intervenciones que contribuyan al mejoramiento del nivel de aprendizaje del ILE. El impacto que esperan tener este tipo de estudios es motivar a los actores antes mencionados para que, en conjunto, reflexionen críticamente sobre dicha problemática con base en los temas, las categorías y los factores identificados en esta investigación y en la literatura nacional; evalúen críticamente los resultados de la actual política nacional del PNB del MEN y procedan a diseñar, implementar y evaluar los resultados de nuevas propuestas que intenten contrarrestar los efectos de los factores externos e internos que están incidiendo en el aprendizaje del inglés y que permitan a los bachilleres obtener unos mejores niveles de AILE. Además, en estas acciones es perentorio que involucren a las autoridades institucionales y municipales para que los apoyen efectivamente, tanto en lo curricular como en lo financiero para asegurar el éxito de sus propuestas.

Por último, en lo administrativo, a las autoridades educativas locales y nacionales se les proporciona información sustentada desde el sector académico, con base en las voces de los propios estudiantes y profesores que viven diariamente la complejidad del fenómeno del aprendizaje del inglés, proceso que se 
encuentra inmerso en las problemáticas del contexto sociocultural de las instituciones educativas, de los hogares de los estudiantes y de las sociedades locales, nacionales e internacionales. El impacto que este estudio aspira a tener en el aspecto administrativo se relaciona con su incidencia en las políticas educativas sobre el aprendizaje del inglés a nivel nacional. Sus resultados deberían motivar a las autoridades educativas de los órdenes municipal, departamental y nacional a evaluar críticamente la efectividad de las políticas del PNB, involucrando a la comunidad académica de la educación primaria, secundaria y superior en la revisión y posible reorientación de dicho programa con el propósito de conformar un currículo articulado. Esta articulación debe buscar una mayor intensidad de estudio del inglés, no solamente como desarrollo de habilidades, sino también como medio de comprensión y producción oral y escrita de conocimientos de las otras áreas disciplinares, especialmente de la educación secundaria y universitaria, de tal manera que el estudio del inglés contribuya al fomento de la interdisciplinariedad en el currículo de la escuela secundaria. Asimismo es necesario que las autoridades educativas adopten un nuevo modelo de toma de decisiones que no se originen exclusivamente en sus dependencias y se impongan a las instituciones educativas (top-down decisions), sino que partan de las bases docentes, estudiantiles y desde los padres de familia (bottomup decisions), para la emisión de las futuras políticas y lineamientos sobre el aprendizaje del inglés, así como también, se apropien de los recursos financieros suficientes para el mejoramiento de la calidad educativa de nuestro país, en general.

Como toda investigación, esta no ha estado exenta de sus limitaciones. En particular, se destaca la dificultad de contar con un mayor número de participantes, especialmente, de estudiantes del grado 11 y de lograr una mayor objetividad en las percepciones de los estudiantes en cada una de las respuestas a las preguntas del cuestionario. Se recomienda la réplica de este tipo de investigaciones en otros departamentos del país y en otros países donde el inglés se enseñe como lengua extranjera. Estos estudios servirán para validar el instrumento, para diseñar nuevos instrumentos de recolección de información, así como también para refutar, corroborar o complementar los resultados de esta investigación.

\section{Referencias}

Alonso, J. C., Díaz, D. M., Martínez, D. A., Mayora, C., Moreno, L. J., y Ochoa, M. J. (2016). Bilingüismo en Santiago de Cali: Análisis de la evolución de los resultados de las Pruebas SABER 11. Cali: Universidad Icesi.

Alsayed, M. (2003). Factors that contribute to success in learning English as a foreign language. Damascus University Journal, 19(1-2), 21-52. Recuperado de http://www.damascusuniversity.edu.sy/mag/human/ images/stories/03230.pdf

Avella, C. P., y Camargo, D. (2010). Exploring student's beliefs about learning English in two public institutions. Cuadernos de Lingüística Aplicada, 15, 76-92

Bastidas, J. A. (1991). EFL in the Colombian high schools: From ivory tower to the poorest high School in Colombia. Lecture presented at the $26^{\text {th }}$ ASOCOPI Convention, Bogotá., D.C.

Bastidas, J. A. (2016). La educación inicial de los profesores de lenguas extranjeras: Un proyecto basado en lineamientos curriculares. Pasto, Colombia: Editorial Universidad de Nariño.

Bastidas, J. A. (2017). More than half a century teaching EFL in Colombia secondary schools: Tracing back our footprints to understand the present. HOW, 24(1), 10-26. http://dx.doi.org/10.19183/ how.24.1.348.

Bastidas, J. A. y Muñoz, G. (2017). La enseñanza del inglés en la educación secundaria en Colombia desde 1972 hasta 1994: Una historia desde los programas, los métodos y los textos. Pasto: Editorial Universidad de Nariño.

Bastidas, J. A. y Muñoz, G. (2018). Hacia una comprensión del bajo nivel de inglés de bachilleres en pasto, Colombia. Ponencia presentada en el X Coloquio Internacional de Investigación en Lenguas Extranjeras. Veracruz, México.

Bastidas, J. A. y Muñoz, G. (Eds). (2011). Fundamentos para el desarrollo Profesional de los profesores de inglés. Pasto: Graficolor Editores.

Batstone, R. (2010). Issues and options in sociocognition. En R. Batstone (Ed.), Sociocognitive perspectives on language use and language learning. Oxford: Oxford University Press. 
Brown, H. D. (2007). Principles of language learning and teaching. Fifth Edition. White Plains. NY: PearsonLongman, Inc.

Cardona, G. y Hanley, W. (1985). Estado actual de la enseñanza del inglés en los colegios de educación básica secundaria y media vocacional de Caldas. Manizales, Caldas: Universidad de Caldas.

Cheng, H. F. y Dörnyei, Z. (2007). The use of motivational strategies in language instruction: The case of EFL teaching in Taiwan. Innovation in Language Learning and Teaching, 1(1), 153-174.

Council of Europe. (2001). Common European framework of reference for languages:

Learning, teaching, assessment. Cambridge: Cambridge University Press.

Dörnyei, Z. (1994). Motivation and motivating in the foreign language classroom. Modern Language Journal, 78, 273-284.

Dörnyei, Z. y Csizér, K. (1998). Ten Commandments for motivating language learners: Results of an empirical study. Language Teaching Research, 2, 203-229.

Eccles, J.S. (2005). Subjective task value and the Eccles et al. Model of achievement-related choices. En A. J. Elliot y C. S. Dweck (Eds.), Handbook of competence and motivation (pp. 105-121). New York: Guilford Press.

Ellis, R. (1994). The study of second language acquisition. Oxford: Oxford University Press.

Ellis, R. (2015). Understanding second language acquisition. Second Edition. Oxford: Oxford University Press.

English First. (2016). EF EPI Proficiency English. Recuperado de https://www.ef.com/ca/epi/reports/epi-s/

English First. (2017). EF EPI Proficiency English. Recuperado de https://www.ef.com/ca/epi/reports/epi-s/

Escalante Angulo, C. (1987). El problema y la hipótesis. Módulo 2. Bogotá: ICFES.

Gardner, R. C. (1985). Social psychology and second language learning: The role of attitudes and motivation. London: Edward Arnold.

Gómez, G. A. (1971). La enseñanza del inglés en Colombia: Su historia y sus métodos. Bogotá, D.E.: Universidad Nacional de Colombia.

Hernández, F. y Faustino, C. C. (2006). Un estudio sobre la enseñanza de lenguas extranjeras en colegios públicos de la ciudad de Cali. Revista Lenguaje, 34, 217-250.
Hidalgo, H. A, y Caicedo, M. L. (2017). La enseñanza del inglés en la educación secundaria en Colombia, el contexto: Una historia desde los programas, los métodos y los textos: 1972-1994. Cali, Colombia: Poemia su Casa Editorial.

ICFES. (2017). Balance: Así les fue a los estudiantes del país en la prueba Saber 11: Icfes. Recuperado de: http://www.icfes.gov.co/en/item/2380.

Johnson, K. E. (2009). Second language teacher education: A socio-cultural perspective. New York: Routledge Taylor \& Francis Group.

Larsen-Freeman, D. y Long, M. (1991). An introduction to second language acquisition research. London, Longman.

León, M. I. (2013). Factors that affect the English language teaching/learning process in Ecuadorian public high school. Tesis de Maestría. Universidad Técnica Particular de Loja, Ecuador.

López, R., Quesada, M. y Salas, J. (2014). Social factors involved in second language learning A case study from the Pacific Campus, Universidad de Costa Rica. Revista de Lenguas Modernas, 21, 435-451. Retrieved from https://revistas.ucr.ac.cr/

Mackey, W. F. (1970). Forward. En L. A. Jakobovits. Foreign Language Learning: A Psycholinguistic analysis of the issues (pp. vii-xiii). Rowley, Mass: Newbury House.

Madrid, D. (1995). Internal and external factors affecting foreign language teaching and learning. Actas de las Segundas Jornadas de Estudios Ingleses. Universidad de Jaén, Jaén, España. (pp. 59-82). Tomado de https:// www.ugr.es/ dmadrid/Publicaciones/Individual\%20 differences.pdf

Madrid, D., Ortega, J., Jiménez, S., Pérez, C., Gomis, A., Fernandez, J.,... y Robinson, R. (1993). Sources of motivation in the EFL Classroom. Actas de las VIII Jornadas Pedagógicas para la Enseñanza del Inglés. GRETA, Granada, España.

Mahmoudi, S. y Mahmoudi, A. (2015). Internal and external factors affecting learning English as a foreign language. International Journal of Language and Linguistics, 3(5), 313-322. doi: 10.11648/j. ijll.20150305.16 
Consejo de Europa. (2001). Marco Común Europeo de Referencia para las Lenguas: Aprendizaje, Enseñanza, Evaluación. Traducido por el Instituto Cervantes. Recuperado de https://cvc.cervantes.es/ensenanza/ biblioteca_ele/marco/

Misbah, N., Mohamad, N., Yunces, M. y Ya’acob, A. (2017). Identifying the factors contributing to students' difficulties in the English Language Learning. Creative Education, 8, 1999/2008.

Morin, E. (1994). Introducción al pensamiento complejo. Barcelona: Gedisa.

Muñoz, C. (2002). Aprender idiomas. Barcelona: Ediciones Paidos, S. A.

Navarrete, A. (1970). La enseñanza de idiomas extranjeros en Colombia. (Unpublished Ph.D. Dissertation). University of Texas, Austin, TX, USA.

Norris, J. M., y Ortega, L. (2000). Effectiveness of L2 instruction: A research synthesis and quantitative meta-analysis. Language Learning, 50, 417-528.

Quesada, P. A., et al. (2017). Teachers' factors that influence the English language teaching-learning process in Ecuadorian high schools in the context of e-education and society. $12^{\text {th }}$ Iberian Conference on Information Systems and Technologies (CISTI). Lisbon, Portugal. DOI: 10.23919/CISTI.2017,7975819. Retrieved from www.ieeexplore.ieee.org

Quintanilla, V. (1971). English in Colombia. ERIC Document. ED 053598.

Richards, J., Platt, J. y Weber, H. (1985). Longman dictionary of applied linguistics. Harlow, Essex: Longman.

Sánchez, A. (2013). Bilingüismo en Colombia. Documentos de Trabajo sobre Economía Regional, 121, 1- 46.

Sanz, C. (Ed.). (2005). Adult second language acquisition. Washington, DC. Georgetown University Press.
Schunk, D. H. y Pintrich, P. R. (2008). Motivation in education: Theory, research, and applications. Upper Saddle River, NJ: Pearson-Merrill Prentice Hall.

Seedhouse, P. (2010). A framework for conceptualizing learning in applied linguistics. In S. Walsh, C. Jenks, y P. Seedhouse (2010). Conceptualizing 'Learning' in applied linguistics. (pp. 240-256). New York: Palgrave Macmillan.

Souriyavongsa, T., Rany, S., Zainol, M., y Mei, L. (2013). Factors cause students low language learning: A case study in the National University of Laos. International Journal of English Language Education, 1(1), 179-192. doi: 10.5296/ijele.v1i1.3100.

Stansfield, C. W. (1972). The teaching of English in Colombian public secondary schools. (Unpublished Ph.D dissertation). The University of Florida, Miami, USA.

Stern, H. H. (1983). Fundamental concepts of language teaching. Oxford: Oxford University Press.

Strevens, P. D. (1977). New orientations in the teaching of English. Oxford: Oxford University Press.

Vygotsky, L. S. (1978). Mind in society. Cambridge, Mass: Harvard University Press.

Wigfield, A. (1994). Expectancy-value theory of achievement motivation: a developmental perspective. Educational Psychology review, 6, 49-78.

Wigfield, A., Tonks, S. y Eccles, J. S. (2004). Expectancy value theory in cross-cultural perspective. En D. M. McInerney y S. Van Etten (Eds.), Big theories revisited (pp. 165-198). Greenwich, CT: Information Age Publishing. 